\title{
Signal Recognition of Polyphase-Coded Radar Signals Based on Multifeature Fusion
}

\author{
Xiaofeng Wang $\mathbb{D}^{\mathrm{D}}$, Huixu Dong, and Ruilan Tian \\ Aviation University of Air Force, Changchun 130022, China \\ Correspondence should be addressed to Xiaofeng Wang; wxf870516@126.com
}

Received 17 December 2021; Revised 10 January 2022; Accepted 12 January 2022; Published 31 January 2022

Academic Editor: Gulam Nabi Alsath

Copyright (c) 2022 Xiaofeng Wang et al. This is an open access article distributed under the Creative Commons Attribution License, which permits unrestricted use, distribution, and reproduction in any medium, provided the original work is properly cited.

\begin{abstract}
For improving recognition accuracy of polyphase-coded radar signals under low signal to noise ratio (SNR), a recognition method based on multifeature and feature selection was proposed. First, feature parameters of polyphase-coded radar signals in time domain, frequency domain, and pseudo-Zernike moments of Choi-Williams distribution (CWD) were extracted, respectively. And then, redundancy features and small-correlation features were removed based on mutual information with greedy idea. Finally, recognition of polyphase-coded radar signals was implemented using support vector machines (SVM). The experimental results showed that the average recognition accuracy of proposed method was over $90 \%$ when SNR is $0 \mathrm{~dB}$, and the recognition performance was superior to the existing methods.
\end{abstract}

\section{Introduction}

Low probability of intercept (LPI) has become a necessary technology for modern radar [1-3]. Polyphase-coded radar signals (Frank code, $P 1$ code, $P 2$ code, $P 3$ code, and $P 4$ code) are widely used in modern radar system due to its good characteristics of LPI [4]. Polyphase-coded radar signals adopts phase encoding to approximate chirp or step chirp for obtaining the advantages of frequency modulation and phase modulation at the same time. It is precisely because the polyphase-coded radar signals has both the characteristics of frequency modulation and phase modulation, and it is difficult to intercept and recognize it, which is a key problem that needs to be solved in electronic intelligence system (ELINT) [5].

For recognition polyphase-coded radar signals in noncooperative electronic reconnaissance conditions, many recognition methods have been proposed. Jennison [6] proposed a neural network polyphase-coded radar signals recognition method; it uses instantaneous characteristics and second-order and third-order statistical characteristics. This method can achieve $97 \%$ recognition accuracy when the SNR is $6 \mathrm{~dB}$. However, when the SNR decreases, the recognition performance is poor. Yuan et al. [7] proposed a polyphase-coded radar signals recognition method based on modulation frequency and component energy ratio; this method can only distinguish the signals into two types simply. Milne and Pace [8] proposed a method for identifying FM continuous wave and $P 4$ code signals based on Wigner distribution. Copeland and Pace [9] solved the same problem based on orthogonal filter banks. But these two methods can only complete the classification of two kinds of signals and have higher requirements for SNR. Lundén and Koivunen [10] constructed a feature vector based on the instantaneous frequency characteristics of the signal and the timefrequency distribution image characteristics to realize polyphase-coded radar signals recognition. Recognition performance of this method depends on the frequency estimation. Deng and Liu [11-13] proposed a polyphasecoded modulation type recognition method based on multiple hypothesis testing. This method is very sensitive to the signal carrier frequency estimation error and the initial phase. Because polyphase-coded radar signals is a carrier frequency suppression signal, it is very difficult to accurately estimate its carrier frequency without prior 
knowledge. Guo et al. [14] proposed a novel recognition algorithm for LPI radar signal based on additive kernel SVM of radar signals complete ensemble empirical mode decomposition with adaptive noise. Zhang et al. [15] proposed LPI radar signal recognition method haled on the stacked autoencoder and SVM. These two methods have high recognition performance at high SNR, but they are not suitable for low SNR. A method based on time-frequency amplitude and phase features can be applied to recognizing polyphase-coded radar signal [16]. For improving recognition performance under the condition of low SNR, a LPI radar signal classification and recognition system based on denoising convolution neural network and inception network is proposed [17]. For reducing the time consumption of the recognition algorithm, $\mathrm{Pu}$ et al. [18] proposed a recognition method based on deep learning convolutional neural network and coordinate transformation of ambiguity function main ridge. However, the prior information required by this method is unknown, and the scalability is not strong. To solve incomplete prior information of radar in noncooperative electronic countermeasure environment, a novel recognition algorithm based on the energy cumulant of CWD is proposed [19].

The difference between polyphase-coded radar signals is very small. The electronic reconnaissance system, especially, works under the condition of low SNR; it is very difficult to recognize coded type of polyphase-coded radar signals. Therefore, a multifeature fusion method for recognizing coded type of polyphase-coded radar signals is proposed. First, multiple signal characteristic parameters are extracted from the time domain, frequency domain, and time-frequency domain to establish an overcomplete recognition feature vector to ensure the accuracy of recognition. Then, based on the mutual information in the information theory, combined with the idea of greed, the feature vector that has little correlation or redundancy with the pattern recognition is eliminated. Finally, SVM classifier is constructed to implement polyphase-coded radar signals recognition.

\section{Signal Model}

The analytical expression of the polyphase-coded radar signals is

$$
x[n]=A e^{j\left(2 \pi f_{c} n+\phi_{m}\right)}, \quad 0 \leq n \leq N-1,
$$

where $A$ is the signal amplitude, $f_{c}$ is the carrier frequency, and $\phi_{m}$ is the phase modulation function. Different phase modulation functions represent different polyphase-coded type. Among polyphase-coded radar signals, Frank, $P 1$, and $P 2$ coded are approximations to the step chirp waveform. The $P 3$ and $P 4$ coded are approximations to the chirp signal at the Nyquist sampling rate. The phase modulation function of each type of polyphase-coded radar signals is shown in Table 1.

\section{Multidomain Feature Extraction}

3.1. Time Domain Feature. According to the definition of polyphase-coded radar signals, essential difference of each code type is its phase modulation function. Therefore, the phase change can be extracted as a characteristic parameter for recognizing polyphase-coded type. The extracted timedomain features include signal instantaneous phase standard deviation and instantaneous frequency standard deviation.

Instantaneous phase standard deviation is defined as

$$
\widehat{\sigma}_{\varphi}=\sqrt{\frac{1}{N}\left(\sum_{n} \varphi^{2}(n)\right)-\left(\frac{1}{N} \sum_{n}|\varphi(n)|\right)^{2}},
$$

where $\varphi(n)$ is the instantaneous phase of the signal without defuzzification, $\varphi(n) \in(-\pi, \pi]$, which is

$$
\varphi(n)=\arg \tan \left\{\frac{\operatorname{Im}[s(n)]}{\operatorname{Re}[s(n)]}\right\} .
$$

Noise will inevitably be mixed into the signal. The randomness of noise makes its impact on different sampling points different. Therefore, when calculating the instantaneous phase, the sampling points that are greatly affected by noise (weak sampling points) should be removed. A large number of simulation experiments show that setting the amplitude threshold to 0.2 of the maximum amplitude can effectively filter out weak sampling points. $N$ is the number of nonweak sampling points.

Instantaneous phase deviation of the signal with respect to time is signal instantaneous frequency. Therefore, instantaneous frequency can be expressed in the form of the first difference of instantaneous phase, that is,

$$
f(n)=\phi_{u}(n+1)-\phi_{u}(n), \quad n=1,2, \ldots, N-1,
$$

where $\phi_{u}(n)$ represents signal instantaneous phase after phase defuzzification. In order to reduce the influence of noise and improve accuracy and stability of instantaneous frequency, multiple phase difference method can be used to extract the instantaneous frequency.

Instantaneous frequency standard deviation is defined as

$$
\begin{aligned}
\widehat{\sigma}_{f} & =\sqrt{\frac{1}{N}\left(\sum_{n} \tilde{f}^{2}(n)\right)-\left(\frac{1}{N} \sum_{n}|\tilde{f}(n)|\right)}, \\
\tilde{f}(n) & =\frac{f(n)-u_{f}}{\max \left|f(n)-u_{f}\right|},
\end{aligned}
$$

where $u_{f}$ is mean value of instantaneous frequency. It should be noted that the process of calculating the instantaneous frequency also needs to remove weak sampling points.

3.2. Frequency Domain Feature. Signal power spectral density can effectively describe energy distribution of signal in frequency domain. Therefore, it is used as a feature for recognizing polyphase-coded radar signals. The extracted frequency domain feature parameters include the maximum power spectrum and the spectrum aggregation measurement value. The maximum power spectrum is defined as 
TABLE 1: Modulation phase of polyphase-coded radar signals.

\begin{tabular}{lc}
\hline Type & $\phi_{m}$ \\
\hline Frank & $\phi_{m}(n, k)=(2 \pi / L)(n-1)(k-1) ; n=1,2, \ldots, L, k=1,2, \ldots, L$ \\
$P 1$ & $\phi_{m}(n, k)=(-\pi / L)[L-(2 k-1)][(k-1) L+(n-1)] ; n=1,2, \ldots, L, k=1,2, \ldots, L$ \\
$P 2$ & $\phi_{m}(n, k)=(-\pi / 2 L)[2 n-1-L][2 k-1-L] ; n=1,2, \ldots, L, k=1,2, \ldots, L(L$ is even $)$ \\
$P 3$ & $\phi_{m}(k)=(\pi / L)(k-1)^{2} ; k=1,2, \ldots, L$ \\
$P 4$ & $\phi_{m}(k)=(\pi / L)(k-1)(k-1-L) ; k=1,2, \ldots, L$ \\
\hline
\end{tabular}

$$
\gamma_{\max }=\frac{1}{N} \max _{k}\left\{\frac{1}{N}\left|\sum_{k=0}^{N-1} \tilde{x}(n) e^{-j 2 \pi n k / N}\right|^{2}\right\}
$$

where $\tilde{x}(n)$ is the signal after amplitude normalization.

Spectrum aggregation measurement value is defined as

$$
C_{M}=\frac{1}{\int_{-\infty}^{\infty}|\overline{X(f)}|^{\alpha} \mathrm{d} f}
$$

where $\alpha \in(0,0.25], \overline{X(f)}$ is the normalized spectrum of signal

$$
\overline{X(f)}=\frac{X(f)}{\int_{-\infty}^{\infty}|X(f)| \mathrm{d} f} .
$$

Polyphase-coded radar signals is essentially a phase modulated signal. The signal will show different feature after being squared. This feature is very beneficial for recognizing signal types. Therefore, the maximum power spectrum value $\gamma_{2, \max }$ and spectrum aggregation measurement value $C_{2, M}$, which is squared signal, are also used as recognizing feature parameter.

3.3. CWD Pseudo-Zernike Moment Feature. CWD has different time-frequency performance and suppresses the cross term by adjusting parameters. The CWD pseudo-Zernike feature has been used to identify the modulation mode of radar signals and has achieved good identification results [19].

CWD of continuous signal $x(t)$ is defined as

$$
W_{\mathrm{CWD}}(t, \omega)=\iint \frac{1}{\sqrt{4 \pi \tau^{2} / \sigma}} e^{\left(-(\mu-t)^{2} / 4 \tau^{2} / \sigma\right)} \cdot x\left(\mu+\frac{\tau}{2}\right) x^{*}\left(\mu-\frac{\tau}{2}\right) e^{-j \omega \tau} d \mu d \tau
$$

where $\sigma(\sigma>0)$ is the scale factor of the CWD. When the scale factor is small, CWD can suppress time-frequency cross-term very well, but the time-frequency resolution will be affected. In order to balance cross-term suppression and resolution improvement, a smaller scale factor is used to suppress the cross-term here. Time-frequency image processing will be used to increase the resolution. Figure 1 is the CWD of Frank coded and P1 coded signal with $\sigma=0.05$.

In order to further reduce cross-term, improve resolution, and reduce influence of parameter settings on the feature, further processing of the CWD image is required. It includes three steps: image thresholding, reducing noise items, and normalizing the aspect ratio. Image thresholding is to obtain a binary image containing only signal components by setting the pixel value threshold, using threshold setting method in literature [20]. The purpose of reducing the interference item is to reduce the influence of strong noise on the CWD image. The open operation in morphology is used to remove the nonsignal components in the binary image.

Pseudo-Zernike moments have rotation, translation, and scale invariance characteristics [21]. These characteristics have been widely used in the field of handwriting recognition. The $p+q$ order origin moment of any digital image $f(x, y)$ is defined as

$$
m_{p q}=\sum_{x} \sum_{y} f(x, y) x^{p} y^{q}
$$

The translational and scale-invariant central geometric moment is defined as

$$
G_{p q}=\frac{1}{m_{00}^{(p+q+2) / 2}} \sum_{x} \sum_{y} f(x, y)(x-\bar{x})^{p}(y-\bar{y})^{q},
$$

where $\bar{x}=\left(m_{10}\right) /\left(m_{00}\right), \bar{y}=\left(m_{01}\right) /\left(m_{00}\right)$. The translational and scale-invariant radial geometric moment is defined as

$$
R_{p q}=\frac{1}{m_{00}^{(p+q+3) / 2}} \sum_{x} \sum_{y} f(x, y)\left(\tilde{x}^{2}+\tilde{y}^{2}\right)^{1 / 2} \widetilde{x}^{p} \tilde{y}^{q},
$$

where $\tilde{x}=x-\bar{x}, \tilde{y}=y-\bar{y}$.

According to the previously mentioned definition, the $n$ order $m$ fold $(|m| \leq n)$ pseudo-Zernike moment of image $f(x, y)$ can be expressed as 


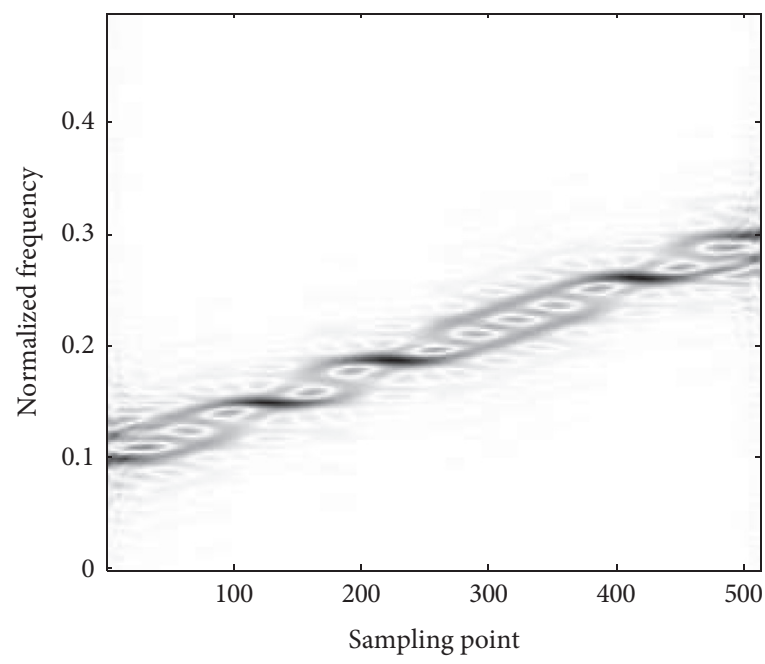

(a)

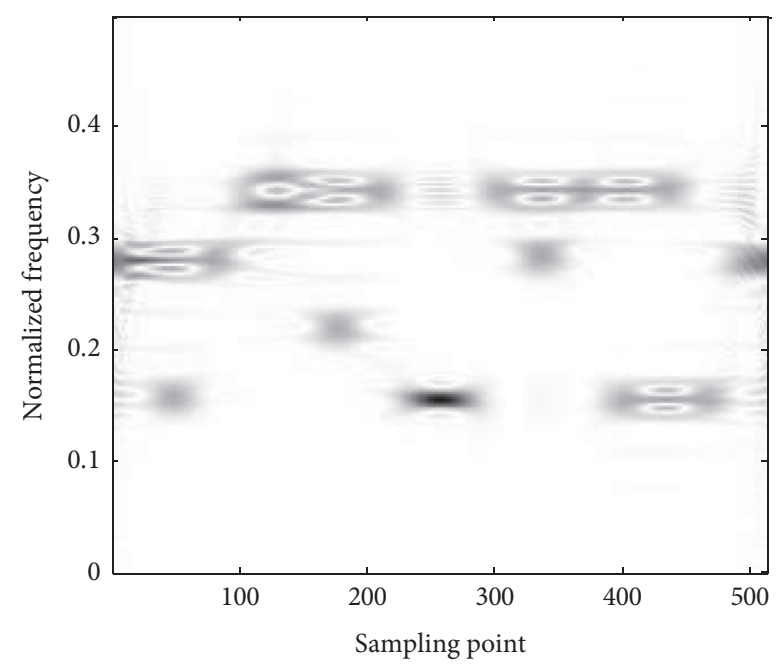

(b)

Figure 1: CWD of polyphase-coded radar signals. (a) Frank coded. (b) $P 1$ coded.

$$
\begin{aligned}
Z_{n m}= & \frac{n+1}{\pi} \sum_{\substack{s=0 \\
n-s-m=\text { even }}}^{n-|m|} D_{n m s} \sum_{a=0}^{k} \sum_{b=0}^{m}(-j)^{b}\left(\begin{array}{l}
k \\
a
\end{array}\right)\left(\begin{array}{l}
m \\
b
\end{array}\right) B_{n m s} G_{2 k-2 a+m-b, 2 a+b} \\
& +\frac{n+1}{\pi} \sum_{\substack{s=0 \\
n-s-m=\text { odd }}}^{n-|m|} D_{n m s} \sum_{a=0}^{d} \sum_{b=0}^{m}(-j)^{b}\left(\begin{array}{l}
d \\
a
\end{array}\right)\left(\begin{array}{l}
m \\
b
\end{array}\right) B_{n m s} R_{2 d-2 a+m-b, 2 a+b},
\end{aligned}
$$

where $k=(n-s-m) / 2, d=(n-s-m-1) / 2$ and

$$
\begin{aligned}
B_{n m s} & =(-1)^{s} \frac{(n-s) !}{s !((n+|m| / 2)-s) !((n+|m| / 2)-s) !}, \\
D_{n m s} & =(-1)^{s} \frac{(2 n+1-s) !}{s !(n+|m|-s) !(n+|m|-s) !}
\end{aligned}
$$

In order to reduce the dynamic range of the characteristic parameters, the logarithm of the pseudo-Zernike moment can be calculated. Therefore, the pseudo-Zernike moment feature used to recognize the polyphase-coded radar signals is

$$
\widehat{Z}_{n m}=\ln \left|Z_{n m}\right| \text {. }
$$

The low-order pseudo-Zernike moments are mainly used to describe the overall characteristics of the image, and the high-order pseudo-Zernike moments are mainly used to describe the local details of the image. The more the loworder moments are, the stronger the noise immunity has. The superior performance of CWD pseudo-Zernike moment features in identifying radar signal modulation types has been demonstrated [22]. The modulation characteristics of polyphase-coded radar signals are highly similar, and electronic reconnaissance is a cooperative environment. Considering the characteristics of high-order moment and low-order moment of pseudo-Zernike moment, the paper determines the selection principle of CWD pseudo-Zernike moment features that combine high-order moment and loworder moment, and low-order moment accounts for the majority. Type recognition experiments are performed for all second to fourth moment features of CWD pseudoZernike moment features. The experimental results show that, $\widehat{Z}_{20}, \widehat{Z}_{22}, \widehat{Z}_{30}, \widehat{Z}_{31}, \widehat{Z}_{32}, \widehat{Z}_{33}$, and $\widehat{Z}_{43}$; these seven CWD pseudo-Zernike moment types have high discrimination for polyphase encoded signals.

In summary, a total of 13 feature parameters for recognizing polyphase-coded radar signals have been extracted. These features include two time domain feature parameters, four frequency domain feature parameters, and seven time-frequency domain feature parameters. Feature parameter list is shown in Table 2.

\section{Feature Selection Based on Mutual Information}

Feature parameter extracted in the time domain, frequency domain, and time-frequency domain may have relatively small or redundant correlations with signal recognition. In order to improve recognition accuracy and efficiency, feature parameter must be filtered. A feature 
TABLE 2: Feature parameter list.

\begin{tabular}{lcc}
\hline $\begin{array}{l}\text { Serial } \\
\text { number }\end{array}$ & Feature name & Symbol \\
\hline 1 & $\begin{array}{c}\text { Instantaneous phase standard deviation } \\
\text { Instantaneous frequency standard } \\
\text { deviation }\end{array}$ & $\widehat{\sigma}_{\varphi}$ \\
2 & $\widehat{\sigma}_{f}$ \\
3 & Maximum power spectrum & $\gamma_{\max }$ \\
4 & Spectrum aggregation measurement & $C_{M}$ \\
5 & Squared maximum power spectrum & $\gamma_{2, \text { max }}$ \\
6 & Squared spectrum aggregation & $C_{2, M}$ \\
7 & measurement & $\widehat{Z}_{20}$ \\
8 & CWD-pseudo-Zernike moments & $\widehat{Z}_{22}$ \\
9 & CWD-pseudo-Zernike moments & $\widehat{Z}_{30}$ \\
10 & CWD-pseudo-Zernike moments & $\widehat{Z}_{31}$ \\
11 & CWD-pseudo-Zernike moments & $\widehat{Z}_{32}$ \\
12 & CWD-pseudo-Zernike moments & $\widehat{Z}_{33}$ \\
13 & CWD-pseudo-Zernike moments & $\widehat{Z}_{43}$ \\
\hline
\end{tabular}

selection method based on mutual information is proposed.

Given a sample data set $T=(O, X, C)$, where $O=\left\{o_{1}, o_{2}\right.$, $\left.\ldots, o_{N}\right\}, X=\left\{x_{1}, x_{2}, \ldots, x_{K}\right\}$ and $C=\left\{c_{1}, c_{2}, \ldots, c_{M}\right\}$ represent sample set, feature parameter set, and category set, respectively. The task of feature selection is to select a set of optimal features from the feature parameter set. In other words, use the least features to effectively classify and reduce data overhead. The key to feature selection is how to remove redundant or unrelated features while retaining useful features.

Entropy is a physical quantity that measures the degree of uncertainty in the value of a random variable. The entropy of any discrete random variable can be defined as

$$
H(X)=-\sum_{x \in X} p(x) \log _{2} p(x)
$$

where $p(x)$ is probability distribution function of random variable. The entropy value is only related to probability density of random variable and has nothing to do with the specific value. To a certain extent, influence of noise and parameter values is avoided. The conditional entropy of two random variables refers to the uncertainty of variable $Y$ when variable $X$ is given as

$$
H(Y \mid X)=-\sum_{x \in X} p(x) \sum_{y \in Y} p(y \mid x) \log _{2} p(y \mid x),
$$

where $p(y \mid x)$ is the conditional probability distribution function. Mutual information describes the content of information shared between two variables, which can measure the degree of interdependence between two random variables. It can be expressed as

$$
I(X ; Y)=-\int_{y} \int_{x} p(x, y) \log _{2} \frac{p(x, y)}{p(x) p(y)} \mathrm{d} x \mathrm{~d} y
$$

where $p(x, y)$ is the joint probability distribution function. When two variables are independent of each other, the mutual information is 0 ; that is, there is no shared information. According to the relationship between mutual information and entropy, mutual information can be expressed in the form of entropy; that is,

$$
\begin{aligned}
I(X ; Y) & =H(Y)-H(Y \mid X) \\
& =H(X)-H(X \mid Y) .
\end{aligned}
$$

Mutual information satisfies symmetry, namely, $I(X ; Y)=I(Y ; X)$.

In process of feature selection, mutual information represents the correlation between category labels and feature parameters; that is,

$$
\begin{aligned}
I(X ; Y)= & H(C)-H(C \mid X) \\
= & -\sum_{c_{i} \in C} p\left(c_{i}\right) \log _{2} p\left(c_{i}\right) \\
& +\int_{X} p(x) \sum_{j=1}^{M} p\left(c_{i} \mid x\right) \log _{2} p\left(c_{i} \mid x\right) \mathrm{d} x,
\end{aligned}
$$

where $M$ is the number of category labels. The first term in equation is the entropy value of the category label, which can be calculated directly based on the sample data, $p\left(c_{j}\right)=N_{j} / N$, where $N_{j}$ is the number of data samples of type $j$ in the sample dataset. Since $x$ is a continuous value, it is impossible to directly calculate the estimation of the posterior probability $p\left(c_{j} \mid x\right)$. The method based on the Parzen window probability density [23] is used to estimate the posterior probability.

The basic idea of the feature selection algorithm is as follows. The final feature selection is achieved through multiple selections using the idea of greed. Only one new feature parameter is selected for one feature selection. This new feature parameter is the feature that satisfies the maximum mutual information with the category set among all the features to be selected. The termination condition of the feature selection algorithm is that the newly added feature parameters make the increment of mutual information less than the specified threshold. The specific steps of the feature selection algorithm are as follows.

(1) Initialization. Let $F$ be the set of $n$ feature parameters to be selected and $S$ be the set of selected feature parameters, $S=\{0\}, f \in F, s \in S$.

(2) Calculate the mutual information between all candidate features and recognition categories $I\left(f_{i} ; C\right)$.

(3) Add the large feature parameter corresponding to the maximum value of mutual information $I\left(f_{i} ; C\right)$ to set $S$, and delete it from set $F$.

(4) the feature selection termination condition is met, go to the next step. If not, return to step (2).

(5) The selected feature set $S$ is used as the output of feature selection, and the feature selection ends.

\section{SVM Classifier Design}

The task of the classifier is to classify the input feature parameter vector into an appropriate category according to a specific criterion. That is to say, the classifier has completed the mapping of the feature space to the category space and 
has completed the classification of the input. The SVM is a classifier based on statistical learning and has achieved good results in many fields. The basic idea of the SVM is to map the input data to a high-dimensional feature space through a nonlinear mapping. Then, implement data classification based on linear classification criteria in a high-dimensional space. Therefore, the SVM is essentially a linear classifier in a high-dimensional space. The specific classification method of SVM is shown in Figure 2.

Traditional SVM is proposed for two classification problems. When solving multiclassification problems, the two-class SVM needs to be extended. At present, there are three main methods for extending traditional SVM: one-toone (OAO) method, one-to-many (OAA) method, and binary tree structure (BTA) method. The existing implementation results show that the performance of $\mathrm{OAO}$ method multiclass extension method is better than OAA method and BTA method. For the recognition of $k$ types of problems, OAO method needs to construct a total of $k(k-$ 1)/2 two-class SVM.

There are five coded types of polyphase-coded radar signals. Therefore, five-class SVM is shown in Figure 3. The classifier has a total of 10 two-class SVM. The input feature parameters first pass through the top-level SVM and then continuously pass into the lower-level SVM. The final recognition result is output by the underlying SVM.

\section{Experiment and Analysis}

Performance of the multifeature fusion recognition algorithm for polyphase-coded radar signals is verified by experiments. The flow diagram of multifeature fusion recognition algorithm is presented in Figure 4.

The normalization method of the input features can be expressed as

$$
x_{i}^{n}=\frac{\left(x_{i}^{n}-\mu_{i}\right)}{\sigma_{i}}, \quad n=1,2, \ldots, N,
$$

where $\mu_{i}$ and $\sigma_{i}$ represent the mean and standard deviation of the $i$-th type features extracted from sample data, respectively. $N$ is the number of sample data.

6.1. Feature Selection Experiment. In feature selection experiment, the sample dataset contains a total of 10000 polyphase-coded radar signals pulses, that is, 2000 pulses for each coding type. The modulation parameters of each coded type are set randomly under standardized constraints. In order to make feature selection robust to noise, the SNR of each pulse is randomly selected from $0 \mathrm{~dB}$ to $20 \mathrm{~dB}$.

The input of the feature selection experiment is 13 feature parameters extracted in the time domain, frequency domain, and time-frequency domain. In the process of feature selection, it is necessary to estimate the mutual information between each feature parameter and the recognition category. If the mutual information between a certain feature parameter and the recognition category is too small, it means that the contribution of this feature parameter to the recognition process is small. In order to reduce the time consumption of the feature selection algorithm, the feature parameters whose mutual information with the classification category is less than the specified threshold will be removed first, and the threshold is set to 0.05 . The number of recognition types is five, and the number of samples in each category is the same, so the entropy value of the first item in the mutual information definition is 2.3219 .

The mutual information value of each step in the feature selection process is shown in Figure 5. The horizontal axis is the serial number of the feature parameter: from left to right indicates the order of selecting the feature parameter. The vertical axis is the mutual information of all selected feature parameters and recognition categories. In order to describe the feature selection process more clearly, the figure shows the mutual information value of all the parameters to be selected. However, in the actual process, when the feature parameter is selected to the seventh, the increment of mutual information is already less than the specified threshold, and the feature selection algorithm has ended. After feature selection, six of the thirteen feature parameters are selected for recognition of the modulation type of the polyphasecoded radar signals.

6.2. Type Recognition Experiment. First, training sample set and test sample set are constructed, respectively. Number of training sample pulses is 500, each type of polyphase-coded radar signals is 100 , and the SNR ranges from $0 \mathrm{~dB}$ to $15 \mathrm{~dB}$. Number of test sample pulses is 1000 , each type of polyphase-coded radar signals is 200 , and the SNR ranges from $-5 \mathrm{~dB}$ to $15 \mathrm{~dB}$. The SNR range of the test sample is larger than the training sample. The purpose is to test recognition algorithm adaptability to noise.

The relationship curve between the recognition accuracy of each signal modulation type and the SNR is shown in Figure 6. Experimental results show that recognition method based on multifeature fusion has higher recognition accuracy for every polyphase-coded radar signals modulation type. When the SNR of the test sample pulse is equal to or better than the training sample, the recognition accuracy rate of each modulation type is greater than $94 \%$, and the overall recognition accuracy rate reaches $95 \%$.

Table 3 shows the confusion matrix for modulation type recognition when the $\mathrm{SNR}$ is $0 \mathrm{~dB}$. The confusion matrix gives the recognition result of each modulation type signal in detail. It can be seen that the error recognition rate between $P 1$ coded and $P 2$ coded, and between Frank coded and P3 coded is relatively high. This is because the modulation characteristics of the two sets of signals are very similar, which can be known from the definition of each modulation type of the polyphase-coded radar signals. In addition, the time-frequency distributions of the two types of signals are also very similar, which makes them easy to misidentify in the identification process.

In order to further verify the performance of the recognition algorithm in this paper, under the same conditions, a simulation comparison experiment was carried out with ensemble empirical mode decomposition and kernel support vector machine (EEMD-KSVM) [14] and stacked 


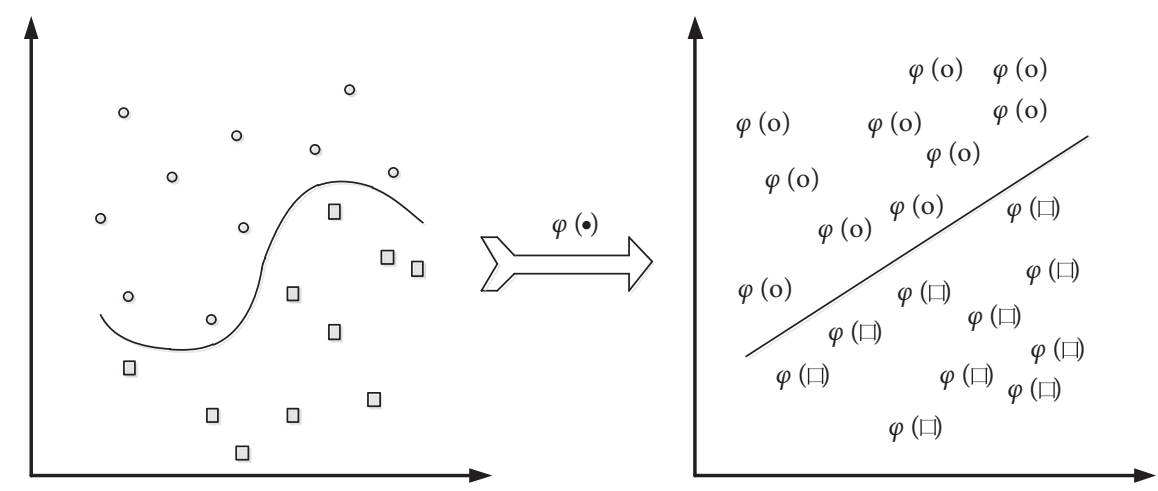

FIGURE 2: Schematic diagram of high-dimensional space mapping.

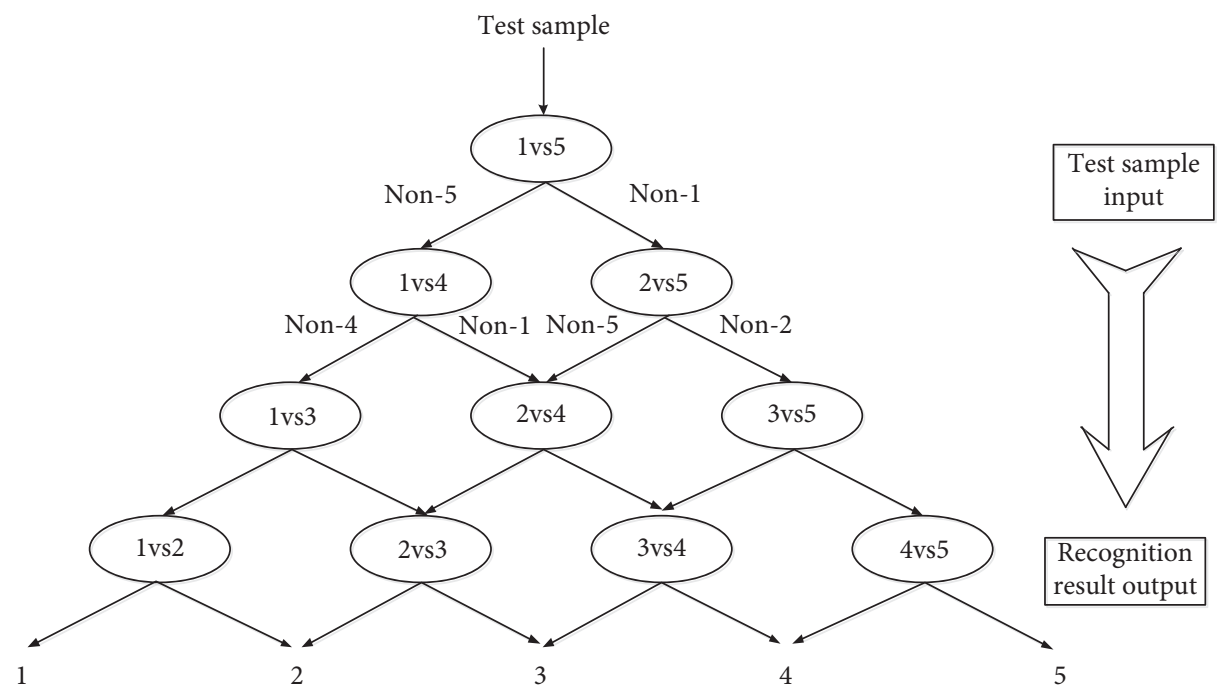

Figure 3: Multiclass SVM.

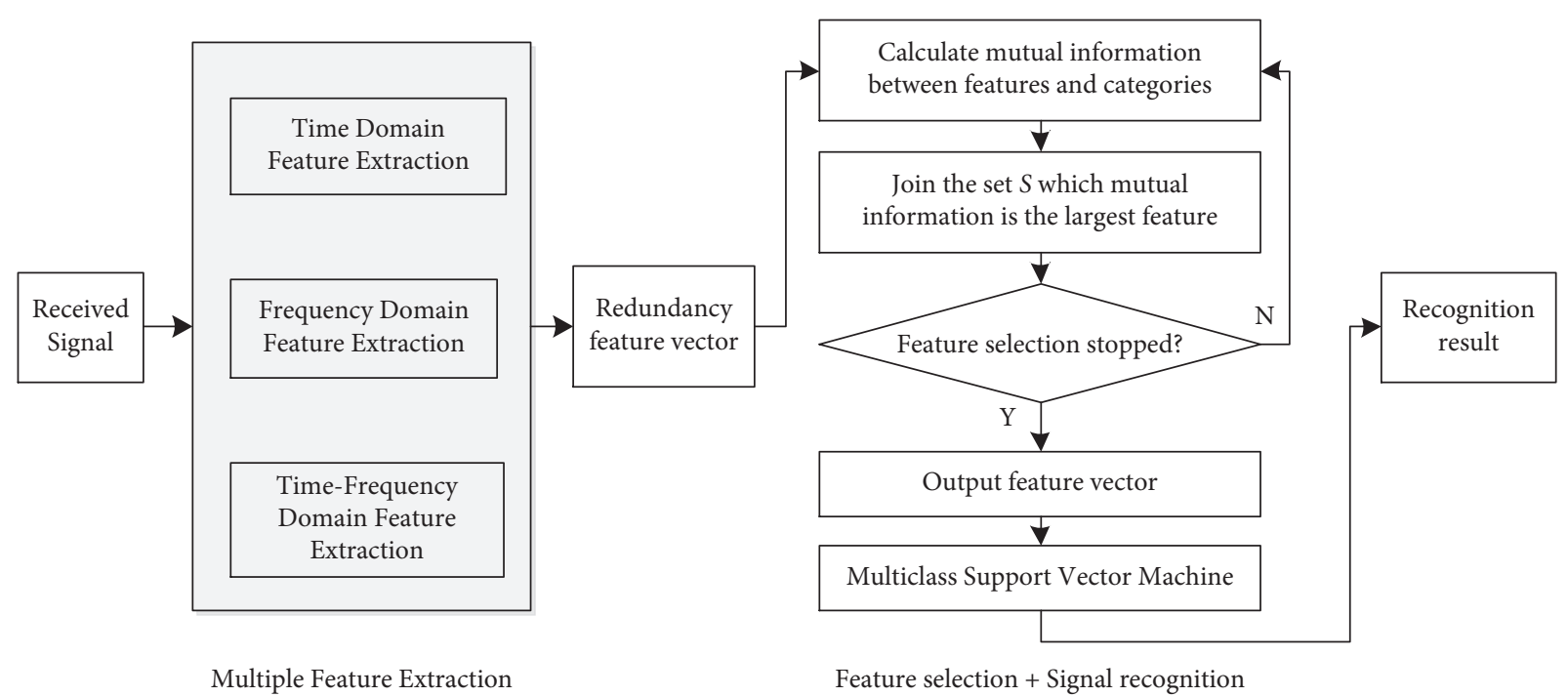

FIgURE 4: Flow diagram of multifeature fusion recognition algorithm.

auto-encoder and support vector machine (SAE-SVM) [15]. The simulation results of three recognition algorithms are shown in Figure 7. Table 4 shows the recognition accuracy of the recognition algorithm in this paper and the other two methods at a SNR of $0 \mathrm{~dB}$. The experimental results show that recognition performance of proposed 


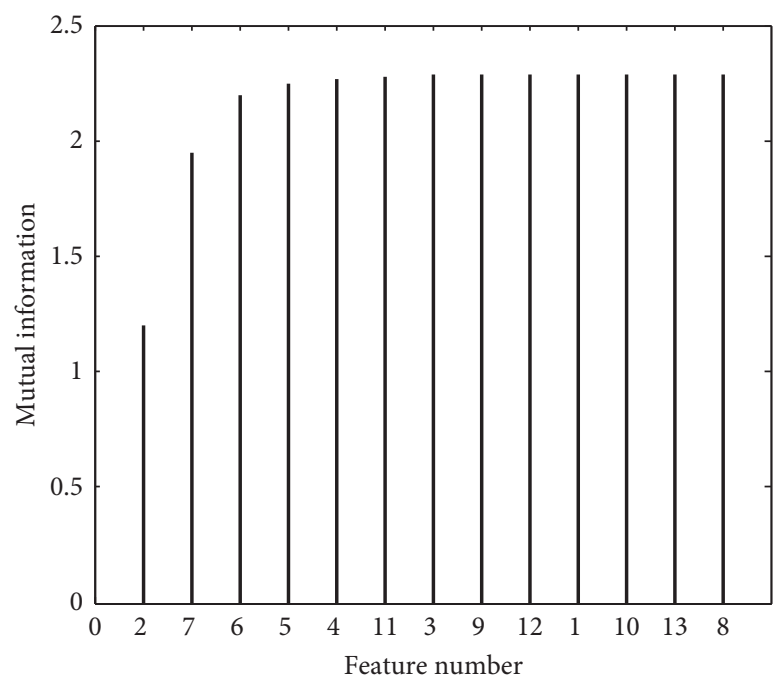

FIgURE 5: Mutual information value in the feature selection process $(H(C)=2.3219)$.

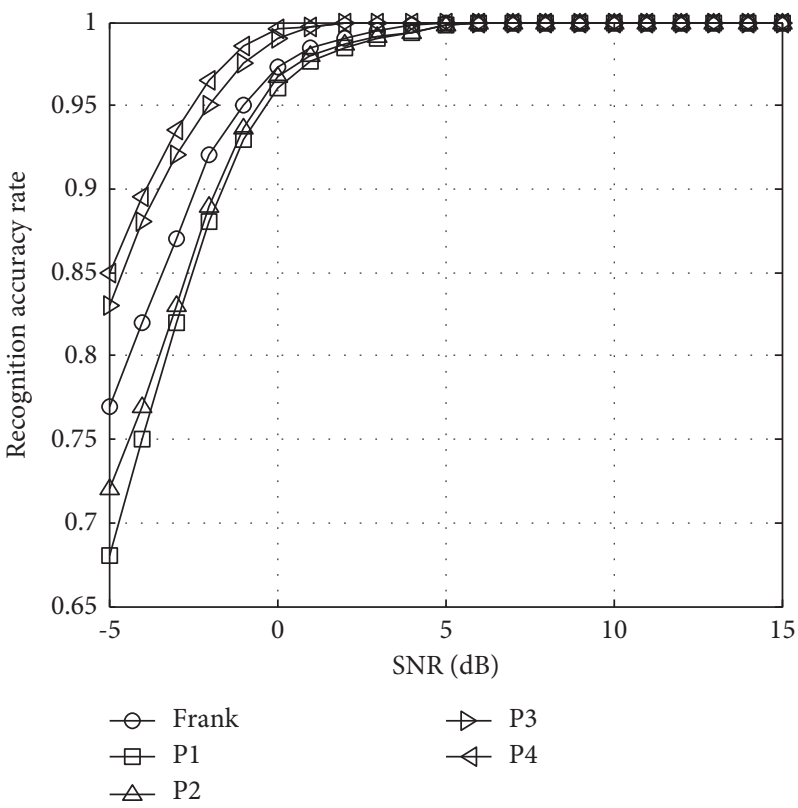

FIgURE 6: Recognition result of every polyphase-coded radar signals modulation type.

TABLE 3: Recognition confusion matrix (\%).

\begin{tabular}{lccccc}
\hline Type & Frank & $P 1$ & $P 2$ & $P 3$ & 0 \\
\hline Frank & 97.3 & 1.4 & 0 & 0 & 1.3 \\
$P 1$ & 1 & 96.0 & 3 & 0 & 0 \\
$P 2$ & 0.9 & 2.4 & 96.7 & 0 \\
$P 3$ & 0 & 0 & 0 & 99.0 & 0.4 \\
$P 4$ & 0 & 0 & 0 & 9.0 \\
\hline
\end{tabular}

algorithm is better than the other two recognition methods for each type of polyphase coded. This is because the proposed algorithm extracts a variety of characteristic parameters, which can characterize signal characteristics. The feature parameter selection is completed based on the mutual information evaluation criterion, and features with excellent performance are selected. The entire recognition process makes full use of the characteristics of each modulation type signal in different "domains," so it has higher recognition accuracy. 


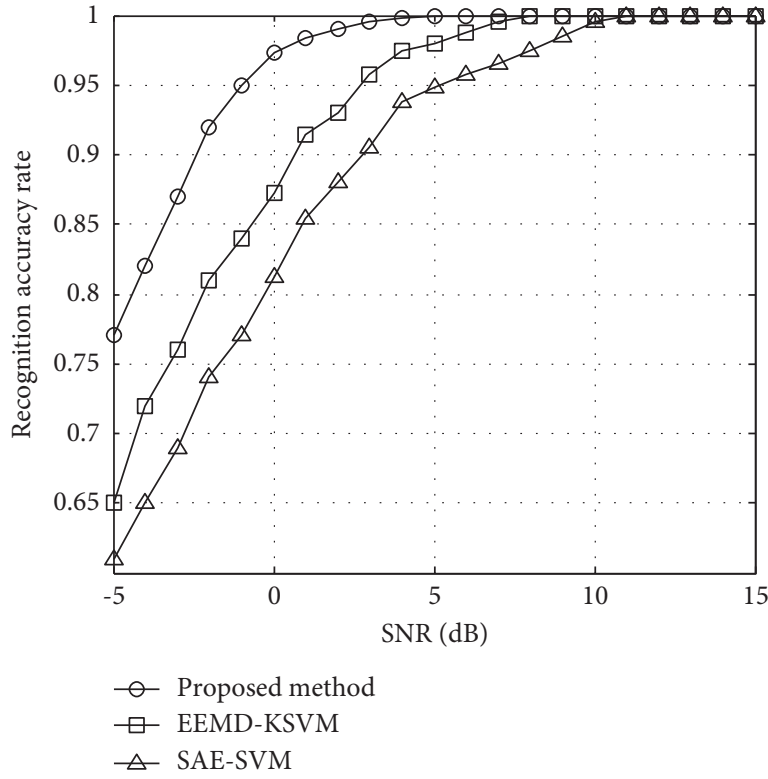

(a)

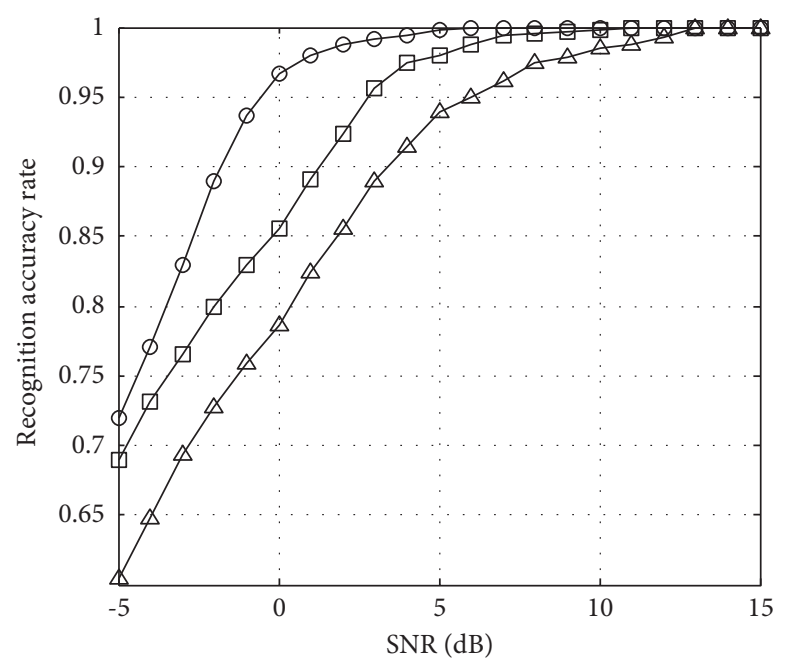

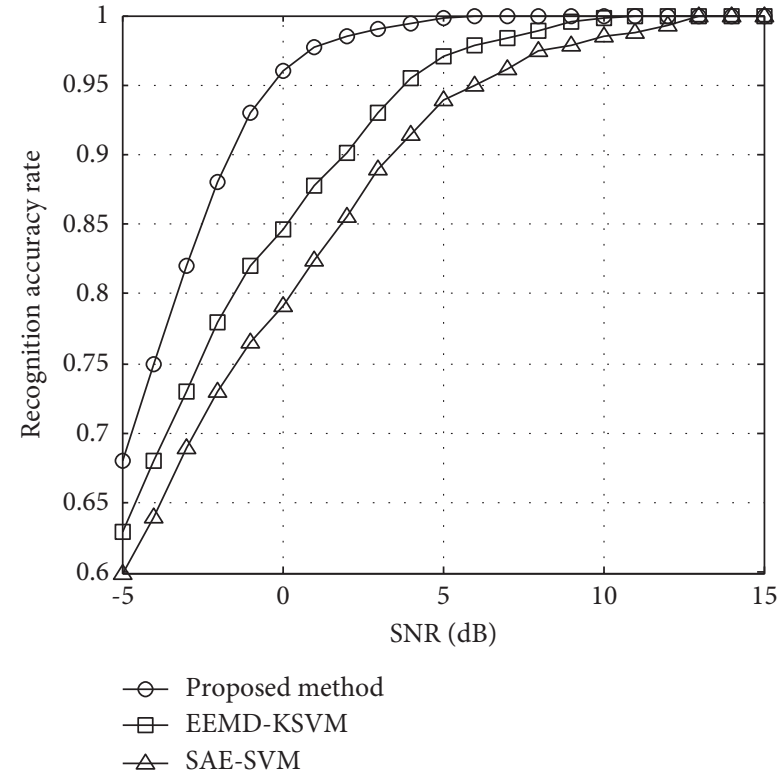

(b)

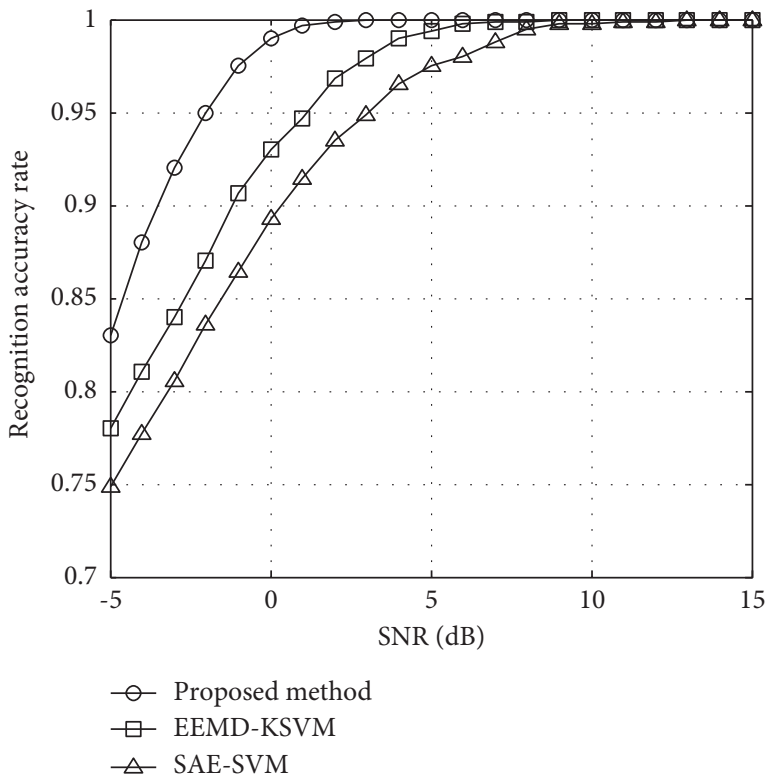

(c)

FIgURe 7: Continued. 


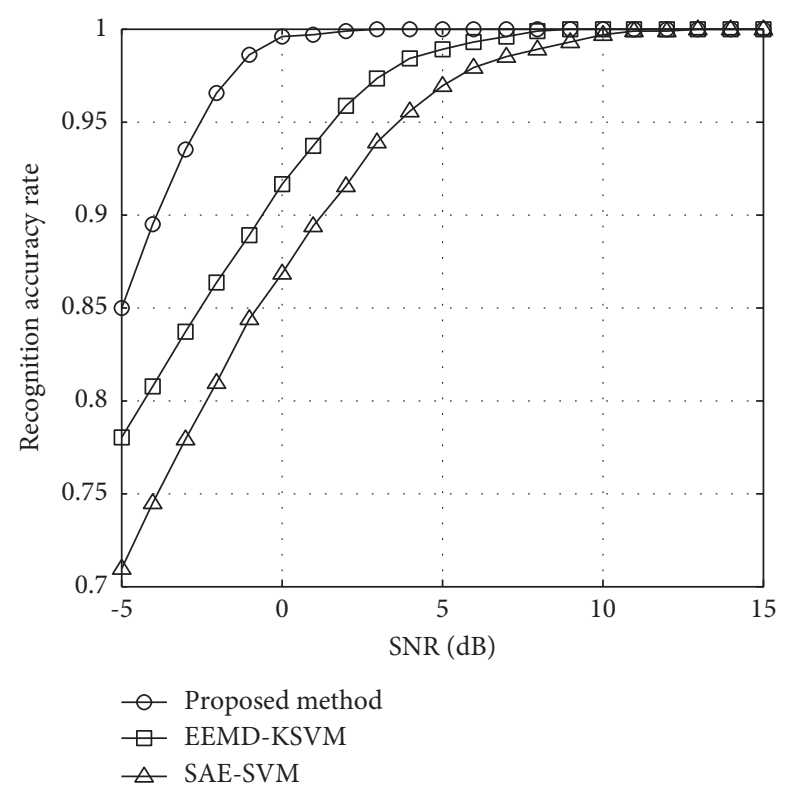

(e)

Figure 7: Comparison of recognition results of three algorithms. (a) Frank code. (b) $P 1$ code. (c) $P 2$ code. (d) $P 3$ code. (e) $P 4$ code.

TABLE 4: Comparison of recognition performance (\%).

\begin{tabular}{lccc}
\hline Type & EEMD-KSVM & SAE-SVM & Proposed method \\
\hline Frank & 87.3 & 81.2 & 97.3 \\
$P 1$ & 84.6 & 79.2 & 96.0 \\
$P 2$ & 89.1 & 78.6 & 96.7 \\
$P 3$ & 93.0 & 89.3 & 99.0 \\
$P 4$ & 92.6 & 86.8 & 99.6
\end{tabular}

\section{Conclusions}

Polyphase-code radar signals recognition method based on multifeature fusion is proposed. First, feature parameters are extracted in time domain, frequency domain, and time-frequency domain. Then, feature selection is implemented based on mutual information. Finally, SVM is used to realize signal recognition. This method can recognize polyphasecoded radar signals modulation type without prior knowledge. Simulation experiments show that the overall recognition accuracy can reach $95 \%$ when the SNR is $0 \mathrm{~dB}$, which is suitable for the recognition of polyphase-coded radar signals in electronic reconnaissance.

\section{Abbreviations}

BTA: Binary tree structure

CWD: Choi-Williams distribution

ELINT: Electronic intelligence system

EEMD- Empirical mode decomposition and kernel

KSVM: $\quad$ support vector machine

LPI: Low probability of intercept

OAO: One-to-one

OAA: One-to-many

SAE-SVM: Stacked auto-encoder and support vector machine
SNR: $\quad$ Signal-to-noise ratio

SVM: $\quad$ Support vector machine.

\section{Data Availability}

The data used to support the findings of this study are included within the article.

\section{Conflicts of Interest}

The authors declare that they have no conflicts of interest.

\section{References}

[1] L. Jiang, L. Li, and G. Q. Zhao, "Polyphase coded low probability of intercept signals detection and estimation using time-frequency rate distribution," IET Signal Processing, vol. 10, no. 1, pp. 46-54, 2016.

[2] M. Wahab, Y. P. Saputera, P. Adhi, and Y. Wahyu, "Development of Indonesian low probability of intercept (LPI) radar system," Defence SeT Technical Bulletin, vol. 10, no. 2, pp. 150-159, 2017.

[3] J. Zhou, F. Wang, M. Sellathurai, and J. Zhou, "Low probability of intercept-based distributed MIMO radar waveform design against barrage jamming in signal-dependent clutter and coloured noise," IET Signal Processing, vol. 13, no. 4, pp. 415-423, 2019.

[4] A. Ragheb, M. A. Esmail, H. Seleem et al., "Photonics-based multi-band/multi-mode radar signal generation," Photonic Network Communications, vol. 39, no. 1, pp. 91-101, 2020.

[5] C. Shi, F. Wang, M. Sellathurai, J. Zhou, and S. Salous, "Low probability of intercept-based optimal power allocation scheme for an integrated multistatic radar and communication system," IEEE Systems Journal, vol. 99, pp. 1-12, 2019.

[6] B. K. Jennison, "Detection of polyphase pulse compression waveforms using the radon-ambiguity transform," IEEE 
Transactions on Aerospace and Electronic Systems, vol. 39, no. 1, pp. 335-343, 2003.

[7] W. Yuan, M. Wang, and S. Wu, "Study on modulation and recognition for LPI radar signals," Signal Processing, vol. 22, no. 2, pp. 153-156, 2006.

[8] P. R. Milne and P. E. Pace, "Wigner distribution detection and analysis of FMCW and P4 polyphase LPI waveforms,"vol. 4, pp. 3944-3942, in Proceedings of the 2002 IEEE International Conference on Acoustics, Speech, and Signal Processing (ICASSP'02), vol. 4, pp. 3944-3942, IEEE, Orlando, FL, USA, May 2002.

[9] D. B. Copeland and P. E. Pace, "Detection and analysis of FMCW and $P 4$ polyphase LPI waveforms using quadrature mirror: filter trees,"vol. 4, pp. 3960-3963, in Proceedings of the 2002 IEEE International Conference on Acoustics, Speech, and Signal Processing (ICASSP'02), vol. 4, pp. 3960-3963, IEEE, Orlando, FL, USA, May 2002.

[10] J. Lundén and V. Koivunen, "Automatic radar waveform recognition," IEEE Journal of Selected Topics in Signal Processing, vol. 1, no. 1, pp. 124-136, 2007.

[11] Z.-M. Deng and Y. Liu, "Recognition and parameters estimation of polyphase-coded radar signals," Journal of Electronics and Information Technology, vol. 31, no. 4, pp. 781-785, 2009.

[12] Z.-M. Deng, L. I. Hai-lin, and Y. Liu, "Likelihood method for recognition of polyphase-coded radar signals," Modern Radar, vol. 31, no. 12, pp. 43-48, 2009.

[13] Z. Deng, Y. Liu, and S. Yang, "Recognition of polyphasecoded radar signals," Journal of Data Acquisition \& Processing, vol. 3, pp. 265-269, 2008.

[14] P. Guo, X. Wang, and Y. Tian, "Low probability of intercept radar signal recognition based on CEEMDAN-ASVM," Modern Radar, vol. 40, no. 3, pp. 27-32, 2018.

[15] M. Zhang, H. Wang, and N. I. Xue, "The LPI radar signal recognition based on deep learning and support vector machine," Science and Technology Review, vol. 37, no. 4, pp. 69-75, 2019.

[16] X. Ni, H. Wang, Y. Yang, Z. Ying, and Z. Zhang, "Polyphasemodulated radar signal recognition based on time-frequency amplitude and phase features," in Proceedings of the International Congress on Image and Signal Processing, BioMedical Engineering and Informatics (CISP-BMEI), Chengdu, China, October 2020.

[17] Y. Xiao, L. Wang, and Y. Guo, "Radar signal modulation type recognition based on denoising convolutional neural network," Journal of Electronics and Information Technology, vol. 43, no. 8, pp. 2301-2307, 2021.

[18] Y. Pu, T. Liu, H. Wu, and G. U. O. Jiang, "Radar emitter signal recognition based on convolutional neural network and coordinate transformation of ambiguity function main ridge," Acta Armamentarii, vol. 42, no. 8, pp. 1680-1689, 2021.

[19] H. Wang, P. Dong, and Y. Chen, "Recognition method of radar signal based on the energy cumulant of choi-williams distribution and improved semi-supervised naïve bayes," Journal of Electronics and Information Technology, vol. 43, no. 3, pp. 590-597, 2021.

[20] H.-I. Choi and W. J. Williams, "Improved time-frequency representation of multicomponent signals using exponential kernels," IEEE Transactions on Acoustics, Speech, \& Signal Processing, vol. 37, no. 6, pp. 862-871, 1989.

[21] R. Keys, "Cubic convolution interpolation for digital image processing," IEEE Transactions on Acoustics, Speech, \& Signal Processing, vol. 29, no. 6, pp. 1153-1160, 1981.
[22] G. Wang, S. Chen, X. Hu, and Y. Junjian, "Radar emitter sorting and recognition based on time-frequency image union feature," in Proceedings of the International Conference on Signal and Image Processing (ICSIP), Wuxi, China, October 2019.

[23] W. Gao, Z. Wei, Y. Luo, and J. Cao, "Artificial bee colony algorithm based on parzen window method," Applied Soft Computing, vol. 74, pp. 679-692, 2019. 